\title{
Percepción del consumo de alcohol propio y de allegados en universitarios de primer curso como predictor del consumo a 10 años
}

\section{Own and others' perceived drinking among freshmen as predictors of alcohol consumption over 10 years}

\author{
Lucía Moure-Rodríguez*, Carina Carbia**, Montserrat Corral Varela***, \\ Ainara Díaz-Geada*, Fernando Cadaveira***, Francisco CaAmaño-Isorna*. \\ * CIBER de Epidemiología y Salud Pública (CIBERESP). Departamento de Medicina Preventiva y Salud Pública. \\ Universidade de Santiago de Compostela. España. \\ ** APC Microbiome Ireland, Biosciences Building, University College Cork, Cork, Irlanda. \\ *** Departamento de Psicología Clínica y Psicobiología. Universidade de Santiago de Compostela. España.
}

\section{Resumen}

El consumo percibido por los jóvenes de sus allegados puede afectar a las expectativas respecto al consumo de alcohol y de este modo, a su propio consumo. El objetivo del estudio ha sido identificar la capacidad predictiva de la percepción de consumo de alcohol propio y de los allegados al inicio del periodo universitario, en los patrones de consumo observados a lo largo de 10 años de seguimiento. Se ha llevado a cabo un estudio de cohortes en universitarios en España ( $\mathrm{n}=1.382)$. Consumo Intensivo de Alcohol (CIA) y Consumo de Riesgo de alcohol (CRA) se midieron con el Test de Identificación de los Trastornos debidos al Uso de Alcohol (AUDIT) a los 18, 20, 22, 24 y 27 años. Se calcularon las Odds Ratios (ORs) con regresión logística multinivel para medidas repetidas. Los universitarios percibían bajo o nulo consumo de alcohol de sus familiares y mayor de sus amigos. Percibir mayor consumo de sus hermanos y amigos aumentó el riesgo de CIA en ambos géneros y de CRA en mujeres. Vivir fuera del domicilio familiar aumentó el riesgo de ambos patrones. En conclusión, la percepción del consumo de alcohol de amigos a los 18-19 años resultó la variable más influyente para el CIA en ambos géneros y el CRA en mujeres a lo largo de 10 años de seguimiento. El consumo de alcohol de los padres al inicio del periodo universitario parece no afectar a los patrones de consumo practicados durante la juventud, una vez se ajusta por el consumo de amigos y hermanos. Vivir en el domicilio familiar actúa como factor protector. Las medidas preventivas en los jóvenes deben tener un enfoque contextual incluyendo a sus allegados.

Palabas clave: Consumo intensivo de alcohol; Amigos; Familia; Jóvenes adultos; Consumo de alcohol en universitarios.

\begin{abstract}
The alcohol use perceptions young people have of those close to them can affect their expectations regarding alcohol and, thus, their own drinking. We aim to identify the predictive ability of own and significant others' perceived drinking at age 18-19 in the alcohol use patterns at 27-28. A cohort study was carried out among university students in Spain ( $\mathrm{n}=1,382)$. Binge Drinking (BD) and Risky Consumption (RC) were measured with the Alcohol Use Disorders Identification Test at ages 18, 20, 22, 24 and 27. Multilevel logistic regression for repeated measures was used to calculate the adjusted Odds Ratios (ORs). College students perceive their family's alcohol consumption as very low or nothing, while the perception of their own alcohol use or that of their friends is higher. Perceiving higher alcohol use among their siblings and friends increases the risk of $\mathrm{BD}$ for both sexes and $\mathrm{RC}$ for women. Living away from the parental home increases the risk of RC and $\mathrm{BD}$. In conclusion, the perception of their friend's alcohol use at age 18-19 is the most influential variable in $\mathrm{BD}$ among both sexes and in RC among men throughout 10 years of follow-up. Parental alcohol consumption does not affect college student drinking patterns when friends and siblings are considered. Living with one's family acts as a protective factor. Preventive measures focused on young people should take a contextual approach and include those closest to them. Keywords: Heavy episodic drinking; Peers; Family; Emerging adulthood; Alcohol drinking in college.
\end{abstract}


$\mathrm{E}$

1 Consumo Intensivo de Alcohol (CIA) ha reemplazado, entre la gente joven, otras formas de consumo más tradicionales en los países de la cuenca mediterránea (Galán, González y Valencia-Martín, 2014; Martinotti et al., 2017). Este patrón se ha definido como la ingesta de grandes cantidades de alcohol en un corto espacio de tiempo, alcanzando unas concentraciones de alcohol en sangre de al menos 0,8 g/1 (Ministerio de Sanidad y Consumo, 2008; National Institute on Alcohol Abuse and Alcoholism, 2004).

Existe una tendencia generalizada a considerar esta forma de consumo como característica de la juventud y del periodo de transición a la edad adulta, tal como ocurre con otras conductas de riesgo (Bava y Tapert, 2010; Chassin, Pitts y Prost, 2002; Crundall, 1995; Schulenberg, O’Malley, Bachman, Wadsworth y Johnston, 1996). Sin embargo, la escasa existencia de estudios longitudinales en Europa sobre el CIA en jóvenes de países mediterráneos no permite confirmar la naturaleza transitoria de esta práctica de consumo. En otros contextos algunos autores han observado como una parte de los jóvenes que practica CIA durante la adolescencia mantienen estos patrones durante la juventud e incluso en los primeros años de la edad adulta (Jefferis, Power y Manor, 2005; Meier, 2010; Patrick et al., 2019).

Nuestro equipo de investigación ha seguido a lo largo de 10 años a una cohorte de jóvenes universitarios, la "Cohorte Compostela”, en la región noroeste de España. Este seguimiento ha permitido constatar que las prácticas de CIA a los 27 años no son tan bajas como se esperaba (Moure-Rodríguez et al., 2016). Estos datos, junto con las consecuencias negativas de estos consumos durante la juventud (Cservenka y Brumback, 2017; Newbury-Birch et al., 2009; World Health Organization, 2018;), evidencian la necesidad de abordar estas conductas.

Los universitarios son una población que presenta elevadas prevalencias de CIA, tanto en nuestra cohorte como en otros estudios (Merrill y Carey, 2016; Moure-Rodríguez et al., 2016; Moure-Rodríguez et al., 2018). Entre las variables que se han relacionado con esta práctica se puede destacar la edad de inicio de consumo, el género y el lugar de residencia (Moure-Rodríguez et al., 2016; Wicki, Kuntsche y Gmel, 2010). Vivir fuera del domicilio familiar o vivir en el campus ha mostrado un mayor riesgo de estas prácticas en estudios previos, principalmente realizados en EEUU, llegando a considerarse el campus como un entorno húmedo, de accesibilidad al alcohol, donde además los pares pueden presentar una mayor influencia (Benz et al., 2020; Simons-Morton et al., 2016), sin embargo los resultados en estudios sobre universitarios en Europa no son tan claros, existiendo diferencias en cuanto al contexto universitario que pueden moldear estas relaciones en distintas direcciones (Kuntsche et al., 2004).

Las expectativas de los jóvenes universitarios respecto al consumo de alcohol a los 18 años han mostrado ser una va- riable explicativa del CRA y el CIA en la Cohorte Compostela 2005 (Moure-Rodríguez et al., 2016; Moure-Rodríguez et al., 2018). La naturaleza modificable de esta variable justifica el interés de la comunidad científica en ella, con investigaciones centradas en modificar las expectativas de los jóvenes, aumentando las expectativas negativas y disminuyendo las positivas, con el objetivo de reducir su consumo de alcohol (Monk y Heim, 2013; Scott-Sheldon, Terry, Carey, Garey y Carey, 2012;). Sin embargo, aunque algunas intervenciones han conseguido modificar las expectativas de los jóvenes respecto al alcohol, esto no ha supuesto cambios en el consumo más allá del primer mes tras la intervención (Scott-Sheldon et al., 2012).

Las expectativas pueden definirse como el conjunto de creencias implícitas o explicitas de un individuo respecto a las consecuencias del consumo de alcohol, a pesar de que existen antes de cualquier experiencia personal de consumo (Miller, Smith y Goldman, 1990). Según Critchlow (1986), se generan probablemente a partir de la observación y de las normas culturales. Como su entorno más cercano y sus principales agentes de socialización durante la infancia, el consumo de alcohol de los padres tendrá una especial influencia en las expectativas y, por tanto, en el consumo de alcohol de los jóvenes (Bahr, Hoffmann y Yang, 2005; Voogt et al., 2017). Aun así, no debemos olvidar que durante la juventud hay una tendencia a estrechar los lazos y socializar con los pares, a medida que se gana autonomía y disminuye el tiempo que se pasa con los padres. Por lo tanto, a estas edades aumenta la influencia de los pares (Brown y Larson, 2009; Patrick y Schulenberg, 2013). Tal como Oei y Morawska (2004) explican, cuando las expectativas -creadas como resultado de los modelos parentales, la influencia de los pares e incluso de los medios de comunicación- se establecen, guían el comportamiento de los jóvenes respecto al consumo de alcohol y, como resultado, este consumo suele confirmar las expectativas.

Steinberg (2014) señaló que a pesar de que los jóvenes tienden a estar en desacuerdo con los padres, suelen coincidir en los asuntos importantes como seguridad y moralidad. Así, tanto el contexto familiar como los pares parecen afectar al consumo de alcohol de los jóvenes (Sellers, McManama, Hernandez y Spirito, 2018; Wood, Read, Mitchell y Brand, 2004), aunque estas relaciones pueden variar en función de la edad o el contexto cultural. La influencia de los pares en el consumo de alcohol se ha abordado con frecuencia, pero apenas existe evidencia del papel de las variables relacionadas con el contexto familiar en población universitaria (Windle, Haardörfer, Lloyd, Foster y Berg, 2017).

Teniendo esto en cuenta, se pretende evaluar cómo la percepción de los universitarios de primer año sobre el consumo de alcohol de sus allegados -madre, padre, hermanos/as, y amigos/as-influye en su consumo de alcohol, no solo durante el primer año de universidad, sino a lo 
largo de 9 años de seguimiento, en nuestro contexto sociocultural y siempre desde una perspectiva de género.

\section{Método}

\section{Diseño, población y muestra}

Se ha realizado un estudio de cohortes entre universitarios (Cohorte Compostela 2005, España) entre noviembre de 2005 y febrero de 2015. Se realizó un muestreo por conglomerados bietápico. Es decir, se seleccionó al menos una clase de primer año de cada una de las 33 facultades (un total de 53 clases) de la Universidade de Santiago de Compostela. El número de clases seleccionadas en cada facultad fue proporcional al número de estudiantes. Todos los estudiantes presentes en el aula el día de la encuesta fueron invitados a participar en el estudio $(n=1.382)$. El 98,6\% de los estudiantes presentes completaron el cuestionario. Los abstemios fueron excluidos del análisis, aunque se presentan en el descriptivo. Los sujetos fueron informados tanto verbalmente como en formato escrito (en el cuestionario) de que la participación era voluntaria, anónima y de la posibilidad de poder abandonar el estudio en cualquier momento sin ningún tipo de consecuencia. Este estudio ha sido aprobado por el Comité de Bioética de la Universidade de Santiago de Compostela.

\section{Recolección de datos}

Dos miembros del equipo de investigación acudieron a cada clase de primer año en noviembre de 2005 e invitaron a todos los estudiantes presentes en el aula a participar en el estudio. En noviembre de 2007 los mismos miembros acudieron a las clases de tercer curso para el seguimiento de los participantes, y llamaron por teléfono a los estudiantes que proporcionaron su número en 2010, 2012 y 2015 (a los 4,5, a los 6,5 y a los 9 años de seguimiento).

En todas las ocasiones se evaluó a los participantes a través de cuestionarios anónimos que se emparejaron cruzándolos a través de las variables fecha de nacimiento, sexo, departamento o facultad y clase.

En las 5 ocasiones se incluyeron los mismos cuestionarios. El consumo de alcohol se midió a través de la versión gallega validada del AUDIT (Saunders, Aasland, Babor, de la Fuente y Grant, 1993; Varela, Carrera, Rial, Braña y Osorio, 2006). Al mismo tiempo se administró otro cuestionario sobre factores potencialmente relacionados con el consumo de alcohol (nivel de educación materna, problemas relacionados con el consumo de alcohol, edad de inicio de consumo, lugar de residencia, expectativas respecto al consumo de alcohol y percepción de consumo propio y de allegados). Para medir las expectativas los participantes debían ordenar catorce expectativas respecto al consumo de alcohol (p. ej. aumenta la diversión, permite olvidar los problemas, produce ansiedad, hace sentirse deprimido), generadas a partir de los ítems de un cuestionario administrado previamente a jóvenes en nuestro país (Defensor del Menor de la Comunidad de Madrid, 2002). Las percepciones se midieron con una escala tipo Likert con 4 categorías (más detalles sobre la recolección de datos en Moure-Rodríguez et al., 2016).

\section{Definición de las variables}

\section{Variables independientes}

Se consideraron las siguientes variables sociodemográficas: género, lugar de residencia (hogar parental/fuera del hogar parental), nivel de educación materna y paterna (primaria/secundaria/universitaria), edad de inicio de consumo de alcohol (mayor de 16 años, a los 16 años, a los 15 años, antes de los 15 años).

Expectativas positivas. Teniendo en cuenta el número de expectativas positivas y negativas se estableció un ranking de 0 a 14 (0 el máximo de expectativas negativas y 14 el máximo de expectativas positivas). Las puntuaciones se clasificaron en terciles, siendo el tercil superior el correspondiente a los sujetos con mayor puntuación en expectativas positivas. Esta variable se dicotomizó codificando el tercil superior como 1 y el tercil medio e inferior como 0 .

Percepción de consumo de alcohol: Percepción del consumo de alcohol propio, de los amigos, hermanos, madre y padre a los 18 años, medido mediante una escala Likert de 4 categorías: nada, poco, bastante o mucho.

\section{Variables dependientes}

1) Consumo de Riesgo de Alcohol (CRA): Variable dicotómica generada a partir de la puntuación total del AUDIT. El punto de corte se estableció en función del género como $\geq 5$ para mujeres y $\geq 6$ para hombres, siguiendo las recomendaciones de la versión gallega validada del AUIDIT (Varela et al., 2006).

2) Consumo Intensivo de Alcohol (CIA). Variable dicotómica generada a partir de la tercera pregunta del AUDIT “¿Con qué frecuencia tomas 6 o más bebidas alcohólicas en una ocasión?", codificada como $0=$ nunca, $0=$ menos de una vez al mes, $1=$ una vez al mes, $1=$ una vez a la semana, $1=$ a diario o casi diariamente. La sensibilidad y especificidad de esta pregunta con este punto de corte es 0,72 y 0,73 respectivamente, y el área bajo la curva de 0,767 (IC 95\%: 0,718 - 0,816) (Tuunanen, Aalto y Seppä, 2007).

\section{Análisis estadístico}

Se realizó regresión logística multinivel para medidas repetidas para obtener las Odds Ratios (ORs) ajustadas por las variables independientes de los modelos finales del CIA y CRA. Se calcularon IC al $95 \%$ para proporciones y medianas. Estos modelos son más flexibles que los tradicionales y por tanto nos permiten trabajar con datos 
interrelacionados. Este es el caso, pues el mismo sujeto se ha medido en diversas ocasiones y, por tanto, las respuestas están fuertemente relacionadas creando una estructura de dependencia. La facultad y la clase fueron consideradas variables de efectos aleatorios. Se decidió no imputar los datos perdidos, pues la distribución de los mismos nos permite asumir que no existen patrones de distribución concretos de los mismos. Se generaron modelos máximos que incluían todas las variables independientes teóricas según la literatura. Los modelos finales se generaron a partir de estos modelos máximos. Las variables no significativas se eliminaron del modelo cuando su exclusión no modificaba más del $10 \%$ los coeficientes de las demás variables y el valor de Akaike Information Criterion (AIC) disminuía. Los datos se analizaron usando los modelos Mixtos Lineales Generalizados con el paquete estadístico SPSS v.20.

\section{Resultados}

Las características de las muestras de mujeres y hombres a los 18 y 19 años se presentan en la Tabla 1 y la Tabla 2. Como se puede observar no existen diferencias estadísticamente significativas para ninguna de las variables en mujeres ni en hombres.

La Tabla 3 presenta las percepciones de consumo propio y de sus allegados, de los participantes. Mientras que solo un tercio de los participantes considera que sus amigos/as consumen poco o nada de alcohol este porcentaje asciende al $68,1 \%$ cuando se refiere al consumo propio y alcanza mayores cifras cuando se refiere a familiares, superando el $90 \%$ en el caso padres y madres. En esta tabla podemos observar la proporción de sujetos con expectativas positivas en función de su percepción de consumo de alcohol. Una mayor proporción de sujetos que perciben el consumo de sus amigos/as, hermanos y/o hermanas, madre o incluso propio como mayor, presentan expectativas positivas respecto al consumo de alcohol, aunque estas diferencias no son estadísticamente significativas.

La Tabla 4 y la Tabla 5 presentan la proporción de sujetos que practican CRA y CIA a los 18, 20, 22, 24 y 27 años en función de la percepción de consumo propio y de sus allegados a los 18-19 años, por separado para mujeres y hombres. Podemos observar una tendencia a una mayor proporción de sujetos que practican ambos patrones de consumo a medida que la percepción de consumo de los allegados aumenta.

Las Figuras 1 y 2 muestran las prevalencias de CRA a los 18, 20, 22, 24 y 27 años según la percepción del consumo propio a los 18 años entre mujeres y hombres respectivamente. Las Figuras 3 y 4 permiten comparar las tendencias de CRA de los jóvenes universitarios de los 18 a los 27 años en función de la percepción de consumo de sus amigos a los 18 años. En todas ellas se puede observar una tendencia general descendente y mayores prevalencias entre los participantes con percepción de consumo propia o de sus amigos elevado a lo largo de los años. Las tablas 6 y 7 presentan los resultados del análisis para CRA y CIA en mujeres y hombres respectivamente, ajustando por las variables incluidas, así como por nivel de educación materna y edad. Las variables nivel de educación paterna y nivel de educación materna no han mostrado asociación con CRA ni CIA.

La percepción de que los amigos/as consumen grandes cantidades de alcohol se relaciona con ambos patrones de consumo en las mujeres $(\mathrm{OR}=17,5$ para el CRA y $\mathrm{OR}=19,3$ para el CIA) y con la práctica de CIA en los hombres $(\mathrm{OR}=17,5)$.

La misma asociación se encuentra en quienes perciben mayor consumo de sus hermanos/as, con hasta tres veces más riesgo de incurrir en CRA en mujeres y 11 veces más en hombres ( $\mathrm{OR}=3,7$ y $\mathrm{OR}=11,6$ respectivamente) y cuatro veces más riesgo de incurrir en CIA en mujeres y el doble en hombres $(\mathrm{OR}=4,5$ y 2,8$)$.

La percepción del consumo de alcohol de su madre no afecta a las prácticas de estos patrones de consumo en las universitarias. Sin embargo, el riesgo de CRA es mayor en el análisis bivariante entre las universitarias que consideran que sus padres consumen poco o bastante alcohol. Esta asociación se invierte si consideran que consumen mucho alcohol. Entre los hombres es la percepción de que sus madres consumen bastante alcohol la que aumenta el riesgo de practicar CRA $(\mathrm{OR}=8,5)$ en el análisis bivariante, la percepción de consumo de alcohol de los padres no se asocia a estas conductas.

Respecto al lugar de residencia, vivir fuera del domicilio familiar aumenta el riesgo de practicar tanto $\mathrm{CRA}(\mathrm{OR}=1,9$ en mujeres y $\mathrm{OR}=1,6$ en hombres) como $\mathrm{CIA}(\mathrm{OR}=1,7$ en mujeres y $\mathrm{OR}=1,6$ en hombres).

\section{Discusión}

Los jóvenes universitarios tienden a percibir que sus madres, padres y hermanos o hermanas consumen poco o nada de alcohol, mientras consideran mayor su propio consumo o el de sus amigos o amigas. A medida que la percepción de consumo de sus allegados aumenta, aumenta también la proporción de sujetos que practican CRA y CIA. Así, el riesgo de practicar CRA y CIA aumenta cuando los universitarios perciben que sus hermanos/as consumen grandes cantidades de alcohol para ambos géneros. Esta asociación también existe respecto a la percepción de consumo de sus amigos, con excepción de la ausencia de riesgo de CRA en hombres. Respecto a la percepción de consumo de sus padres, solamente se asocia con un aumento de CRA entre las mujeres, mientras que la percepción de consumo de las madres se asocia a un mayor riesgo de CRA entre los hombres, ambos en el análisis bivariante. Por último, vivir fuera del domicilio familiar aumenta el riesgo de ambos patrones de consumo tanto en hombres como en mujeres. 
Tabla 1. Características de las mujeres al inicio del estudio en la muestra inicial y durante el seguimiento.

\begin{tabular}{|c|c|c|c|c|c|c|}
\hline & \multicolumn{5}{|c|}{ Porcentaje o media $(95 \% \mathrm{Cl})$} & \multirow[b]{2}{*}{ p-valor } \\
\hline & $\begin{array}{c}\text { Inicial } \\
\text { (18-19 años) }\end{array}$ & $\begin{array}{c}\text { 2-años de } \\
\text { seguimiento } \\
\text { (20-21 años) }\end{array}$ & $\begin{array}{c}\text { 4-años de } \\
\text { seguimiento } \\
\text { (22-23 años) }\end{array}$ & $\begin{array}{c}\text { 6-años de } \\
\text { seguimiento } \\
\text { (24-25 años) }\end{array}$ & $\begin{array}{l}\text { 9-años de } \\
\text { seguimiento } \\
\text { (27-28 años) }\end{array}$ & \\
\hline & $n=992$ & $n=669(67,4 \%)$ & $n=461(46,5 \%)$ & $n=266(26,8 \%)$ & $n=325(32,8 \%)$ & \\
\hline \multicolumn{7}{|c|}{ Nivel de educación materna } \\
\hline Secundaria & $33,6(30,2-37,1)$ & $30,5(26,4-34,7)$ & $30,6(25,8-35,8)$ & $26,5(20,4-33,3)$ & $28,1(22,5-34,2)$ & \\
\hline Universitaria & $24,6(21,2-28,1)$ & $25,3(21,3-29,6)$ & $26,3(21,4-31,4)$ & $26,1(20,1-32,9)$ & $26,2(20,7-32,4)$ & 0,642 \\
\hline \multicolumn{7}{|c|}{ Edad de inicio de consumo } \\
\hline Mayor de 16 años & $19,0(16,5-21,8)$ & $17,9(14,9-21,3)$ & $16,5(13,0-20,5)$ & $16,7(12,1-22,5)$ & $14,5(10,5-19,2)$ & \\
\hline A los 16 años & $38,9(35,6-42,2)$ & $38,1(34,1-42,2)$ & $36,8(32,0-41,7)$ & $40,1(33,6-46,8)$ & $36,6(30,9-42,6)$ & \\
\hline Antes de los 15 años & $16,5(14,0-19,7)$ & $18,1(15,0-21,5)$ & $20,3(16,4-24,5)$ & $16,7(12,1-22,5)$ & $20,7(16,0-25,9)$ & 0,438 \\
\hline AUDIT (mediana) & $5,4(5,2-5,7)$ & $5,6(5,1-5,8)$ & $5,6(5,2-6,0)$ & $5,6(5,0-6,1)$ & $5,3(4,9-5,8)$ & 0,884 \\
\hline \multicolumn{7}{|c|}{$\begin{array}{l}\text { Percepción del consumo de alcohol } \\
\text { propio a los 18-19 }\end{array}$} \\
\hline No consumo & 19,1 & 19,1 & 18,2 & 20,3 & 19,7 & \\
\hline Consumo poco & 53,7 & 53,2 & 54,0 & 54,9 & 54,5 & \\
\hline Consumo bastante & 23,1 & 23,2 & 23,2 & 20,7 & 22,5 & \\
\hline Consumo mucho & 3,2 & 3,4 & 3,5 & 3,8 & 2,8 & 0,765 \\
\hline
\end{tabular}

Tabla 2. Características de los hombres al inicio del estudio en la muestra inicial y durante el seguimiento.

\begin{tabular}{|c|c|c|c|c|c|c|}
\hline & \multicolumn{5}{|c|}{ Porcentaje o media (IC 95\%) } & \multirow[b]{2}{*}{ p-valor } \\
\hline & $\begin{array}{l}\text { Inicial } \\
\text { (18-19 años) }\end{array}$ & $\begin{array}{c}2 \text { años de } \\
\text { seguimiento } \\
\text { (20-21 años) }\end{array}$ & $\begin{array}{c}\text { 4-años de } \\
\text { seguimiento } \\
\text { (22-23 años) }\end{array}$ & $\begin{array}{c}\text { 6-años de } \\
\text { seguimiento } \\
\text { (24-25 años) }\end{array}$ & $\begin{array}{c}\text { 9-años de } \\
\text { seguimiento } \\
\text { (27-28 años) }\end{array}$ & \\
\hline & $\mathrm{n}=\mathbf{3 7 1}$ & $\mathrm{n}=206(55,5 \%)$ & $\mathrm{n}=139(37,5 \%)$ & $n=81(21,8 \%)$ & $n=90(24,2 \%)$ & \\
\hline \multicolumn{7}{|c|}{ Nivel de educación materna } \\
\hline Secundaria & $27,6(22,1-33,3)$ & $27,4(19,9-34,9)$ & $25,5(16,8-34,7)$ & $24,1(12,7-35,8)$ & $27,0(16,8-38,9)$ & \\
\hline Universitaria & $40,3(34,8-46,0)$ & $36,8(29,3-44,3)$ & $32,8(24,1-42,0)$ & $32,9(21,5-44,7)$ & $31,5(21,3-43,4)$ & 0,449 \\
\hline \multicolumn{7}{|c|}{ Edad de inicio de consumo } \\
\hline Mayor de 16 años & $18,1(12,5-24,1)$ & $16,8(9,2-24,7)$ & $15,5(6,9-25,5)$ & $16,4(6,0-29,7)$ & $18,2(7,8-30,3)$ & \\
\hline A los 16 años & $36,9(31,2-42,8)$ & $41,0(33,5-49,0)$ & $44,0(35,3-54,0)$ & $50,7(40,3-64,0)$ & $48,1(37,7-60,1)$ & \\
\hline Antes de los 15 años & $23,4(17,8-29,4)$ & $22,0(14,4-30,0)$ & $19,0(10,3-9,0)$ & $9,0(0,0-22,3)$ & $13,0(2,6-25,1)$ & 0,381 \\
\hline AUDIT (mediana) & $7,8(7,2-8,4)$ & $7,4(6,6-8,2)$ & $7,3(6,4-8,2)$ & $6,5(5,4-7,6)$ & $7,1(6,0-8,2)$ & 0,784 \\
\hline \multicolumn{7}{|c|}{$\begin{array}{l}\text { Percepción del consumo de alcohol } \\
\text { propio a los 18-19 }\end{array}$} \\
\hline No consumo & 16,2 & 18,9 & 20,1 & 19,8 & 17,8 & \\
\hline Consumo poco & 39,4 & 40,3 & 41,7 & 45,7 & 42,2 & \\
\hline Consumo bastante & 33,7 & 30,6 & 29,5 & 27,2 & 30,0 & \\
\hline Consumo mucho & 9,4 & 9,2 & 8,6 & 7,4 & 10,0 & 0,830 \\
\hline
\end{tabular}

La elevada participación alcanzada en este estudio (un 98,6\% del alumnado presente en clase en el primer contacto) nos permite valorar muy positivamente la representatividad de la muestra y por tanto los resultados obtenidos.
La participación a lo largo del seguimiento se vio reducida, con 1.363 sujetos en el primer contacto, 875 a los 2 años de seguimiento, 600 a los 4 años de seguimiento, 347 a los 6 años de seguimiento y 415 a los 9 años de seguimiento. 
Tabla 3. Porcentaje de mujeres y hombres con expectativas positivas respecto al alcohol en función de su percepción de consumo de alcohol propio, y de sus allegados.

\begin{tabular}{|c|c|c|}
\hline & \multicolumn{2}{|c|}{ Expectativas positivas (\%) } \\
\hline & Mujeres & Hombres \\
\hline & $\mathrm{n}=992$ & $n=371$ \\
\hline \multicolumn{3}{|l|}{$\begin{array}{l}\text { Percepción del consumo } \\
\text { de alcohol propio }\end{array}$} \\
\hline No consumo $(18,3 \%)$ & 13,7 & 14,3 \\
\hline Consumo poco $(49,8 \%)$ & 25,5 & 24,8 \\
\hline Consumo bastante $(25,9 \%)$ & 46,3 & 42,4 \\
\hline Consumo mucho $(5,0 \%)$ & $50,0^{*}$ & $55,2^{*}$ \\
\hline \multicolumn{3}{|l|}{ Perdidos (1,0\%) } \\
\hline \multicolumn{3}{|l|}{$\begin{array}{l}\text { Percepción del consumo } \\
\text { de alcohol de amigos/as }\end{array}$} \\
\hline No consumen $(1,8 \%)^{\mathrm{a}}$ & 11,8 & 0,0 \\
\hline Consumen poco $(29,3 \%)$ & 20,8 & 25,8 \\
\hline Consumen bastante $(48,1 \%)$ & 32,1 & 34,3 \\
\hline Consumen mucho $(19,9 \%)$ & $36,8^{*}$ & 36,4 \\
\hline \multicolumn{3}{|l|}{ Perdidos (o,8\%) } \\
\hline \multicolumn{3}{|l|}{$\begin{array}{l}\text { Percepción del consumo de } \\
\text { alcohol de los hermanos/as }\end{array}$} \\
\hline No consumen $(44,9 \%)$ & 27,4 & 34,2 \\
\hline Consumen poco $(28,2 \%)$ & 26,6 & 31,8 \\
\hline Consumen bastante $(12,8 \%)$ & 41,6 & 25,6 \\
\hline Consumen mucho $(2,7 \%)$ & $44,4^{\star}$ & $77,8^{\star}$ \\
\hline \multicolumn{3}{|l|}{ Perdidos (11,5\%) } \\
\hline \multicolumn{3}{|l|}{$\begin{array}{l}\text { Percepción del consumo } \\
\text { de alcohol de la madre }\end{array}$} \\
\hline No consume $(62,2 \%)$ & 28,7 & 33,7 \\
\hline Consume poco $(35,5 \%)$ & 28,7 & 29,8 \\
\hline Consume bastante $(0,7 \%)$ & 60,0 & 40,0 \\
\hline Consume mucho $(0,2 \%)$ & - & - \\
\hline \multicolumn{3}{|l|}{ Perdidos $(1,3 \%)$} \\
\hline \multicolumn{3}{|l|}{$\begin{array}{l}\text { Percepción del consumo } \\
\text { de alcohol del padre }\end{array}$} \\
\hline No consume $(32,4 \%)$ & 28,4 & 35,4 \\
\hline Consume poco $(57,7 \%)$ & 29,7 & 32,4 \\
\hline Consume bastante $(6,6 \%)$ & 28,1 & 23,1 \\
\hline Consume mucho $(1,0 \%)$ & 14,3 & 20,0 \\
\hline Perdidos $(2,2 \%)$ & & \\
\hline
\end{tabular}

Nota.

a Porcentajes de sujetos en la categoría de hombres y mujeres combinados.

* Diferencias significativas entre categorías de exposición. Test $X^{2}$, p<0.05.

Sin embargo, como se puede observar en las tablas 1 y 2 no ha habido diferencias estadísticamente significativas en las características iniciales de los participantes a lo largo de los 9 años de seguimiento, por lo que se puede considerar que la representatividad de la muestra inicial no se ha perdido.

Más de la mitad de los participantes considera que sus amigos consumen bastante o mucho alcohol, lo que va en línea con las elevadas prevalencias de consumo en jóvenes referidas en nuestro país (Ministerio de Sanidad, 2018a; Ministerio de Sanidad, 2018b) así como en otros países -con uno de cada 3 jóvenes europeos practicando CIA mensualmente- (ESPAD Group, 2016; Farke y Anderson, 2007). A este respecto, la prevalencia de CIA en la Cohorte Compostela a los 18 años es $17,9 \%$ y $35,6 \%$ para mujeres y hombres respectivamente. Además, los universitarios suelen consumir más alcohol y de un modo más intenso que sus pares no universitarios (Center for Behavioral Health Statistics and Quality, 2015; Merrill y Carey, 2016; Quinn y Fromme, 2011;). De todos modos, no debemos olvidar que los jóvenes, y en concreto los universitarios, tienden a sobreestimar el consumo de sus pares y/o amigos (Cox et al., 2019; Dumas, Davis y Neighbors, 2019), por tanto, nuestros datos pueden estar en parte reflejando esta sobreestimación.

La importante diferencia entre las percepciones de su propio consumo y las de sus amigos observada (Tabla 3), puede estar influida también por una tendencia a infraestimar el propio consumo. En este sentido Gual et al. (2017) encontraron que hasta el 93,7\% de los bebedores de riesgo no se ven como consumidores excesivos. Los datos que se presentan en las Figuras 1 y 2 parecen avalar esta infraestimación pues un $35,9 \%$ de las mujeres y un $27,2 \%$ de los hombres que declararon no consumir alcohol o consumir poco alcohol muestran CRA. Además, casi todos los participantes que declaran consumir bastante alcohol o mucho alcohol a los 18 años muestran CRA a esta misma edad $-95,0 \%$ de las mujeres; $96,9 \%$ de los hombres-, por tanto, entre los jóvenes que consideran que consumen bastante o mucho alcohol no parece estar existiendo una sobreestimación del consumo propio.

El elevado porcentaje de participantes que consideran que sus hermanos/as consumen poco o nada de alcohol $-73,1 \%$ - puede deberse en parte a que sean hermanos o hermanas menores. La falta de información a este respecto no permite realizar más consideraciones.

Respecto al consumo de las madres, prácticamente el 98\% de los participantes consideran que sus madres no consumen alcohol o consumen poco alcohol, por tanto, casi no existe variabilidad en las respuestas. Estos datos pueden ser un reflejo parcial de los patrones de consumo tradicionales practicados por los hombres (Galán et al., 2014; Wilsnack, Wilsnack y Obot, 2005), aunque las diferencias de género en muchos países están disminuyendo entre las generaciones más jóvenes (ESPAD Group, 2016; Wilsnack et al., 2005).

Las percepciones de consumo de alcohol de los padres son mayores que las de las madres, pero menores que las de consumo propio o de los amigos, y que las prevalencias de consumo de alcohol entre la población española, que ronda el 63\% (Ministerio de Sanidad, 2018b).

Las bajas percepciones de consumo de los progenitores pueden deberse a una normalización del mismo pues, 
Tabla 4. Porcentajes de mujeres que practican consumo de riesgo y consumo intensivo de alcohol entre los 18 y los 27 años en función de su percepción de consumo propio y de sus allegados a los 18-19.

\begin{tabular}{|c|c|c|c|c|c|c|c|c|c|c|}
\hline & \multicolumn{5}{|c|}{ Consumo de Riesgo de Alcohol (\%) } & \multicolumn{5}{|c|}{ Consumo Intensivo de Alcohol (\%) } \\
\hline & \multicolumn{5}{|c|}{ Edad } & \multicolumn{5}{|c|}{ Edad } \\
\hline & 18 & 20 & 22 & 24 & 27 & 18 & 20 & 22 & 24 & 27 \\
\hline \multicolumn{11}{|c|}{ Percepción de consumo propio } \\
\hline No consumo & 1,6 & 8,6 & 4,8 & 1,9 & 3,1 & 0,0 & 1,6 & 1,2 & 1,9 & 0,0 \\
\hline Consumo poco & 48,0 & 49,0 & 42,6 & 11,0 & 18,1 & 9,9 & 9,6 & 12,0 & 5,5 & 1,7 \\
\hline Consumo mucho & $100^{*}$ & $100^{*}$ & $81,2^{\star}$ & $10,0^{*}$ & 66,7 & $84,4^{*}$ & $78,3^{*}$ & 56,2 & 0,0 & $44,4^{\star}$ \\
\hline \multicolumn{11}{|c|}{ Percepción del consumo de alcohol de los amigos/as } \\
\hline No consumen & 5,0 & 5,3 & 8,3 & 0 & 11,1 & 0,0 & 5,3 & 0,0 & 0,0 & 0,0 \\
\hline Consumen poco & 28,1 & 31,7 & 31,9 & 8,0 & 9,3 & 4,6 & 8,3 & 7,2 & 3,4 & 0,0 \\
\hline Consumen bastante & 62,2 & 60,1 & 46,8 & 12,9 & 23,8 & 20,3 & 15,3 & 18,2 & 4,5 & 4,3 \\
\hline \multicolumn{11}{|c|}{ Percepción del consumo de alcohol de hermanos/as } \\
\hline No consumen & 44,5 & 42,2 & 37,8 & 8,7 & 12,1 & 13,3 & 11,6 & 11,9 & 2,4 & 2,1 \\
\hline Consumen poco & 47,0 & 51,8 & 42,5 & 12,5 & 23,3 & 15,7 & 16,9 & 17,3 & 4,7 & 7,0 \\
\hline Consumen bastante & 76,2 & 72,6 & 58,1 & 22,2 & 42,2 & 30,2 & 28,4 & 21,0 & 8,3 & 4,4 \\
\hline Consumen mucho & $87,0^{*}$ & $82,4^{\star}$ & $81,2^{*}$ & 10,0 & $37,5^{*}$ & $52,2^{\star}$ & $41,2^{\star}$ & $25,0^{\star}$ & 10,0 & 37,5 \\
\hline \multicolumn{11}{|c|}{ Percepción del consumo de alcohol de la madre } \\
\hline No consume & 51,6 & 53,1 & 43,1 & 11,2 & 18,0 & 17,1 & 15,7 & 16,2 & 4,5 & 3,4 \\
\hline Consume poco & 51,6 & 50,2 & 42,2 & 12,0 & 25,9 & 19,1 & 19,3 & 13,6 & 3,6 & 7,1 \\
\hline Consume bastante & 80,0 & 75,0 & 100 & 50,0 & 33,3 & 0 & - & $66,7^{\star}$ & & - \\
\hline Consume mucho & - & - & - & - & - & - & - & - & - & - \\
\hline \multicolumn{11}{|c|}{ Percepción del consumo de alcohol del padre } \\
\hline Consume bastante & 55,7 & 65,9 & 50,0 & 9,5 & $38,7^{\star}$ & 19,7 & 27,3 & 14,7 & 0,0 & 9,7 \\
\hline Consume mucho & $12,5^{\star}$ & 20,0 & $0,0^{\star}$ & - & - & 12,5 & 0,0 & - & - & - \\
\hline Total de sujetos & 51,5 & 52,2 & 43,2 & 12,2 & $20,9^{\wedge}$ & 17,9 & 16,7 & 15,7 & 4,1 & $4,9^{\wedge}$ \\
\hline
\end{tabular}

siendo los principales agentes sociales durante la infancia (Voogt et al., 2017), es fácil asumir sus costumbres como referente de normalidad, a pesar de las recomendaciones internacionales de salud. Por otra parte, su forma de consumo, además de ser la habitual en el domicilio, seguramente es la tradicional en nuestro contexto social y cultural -consumo regular acompañando a las comidas (Galán et al., 2014; Willett et al., 1995)- y probablemente diferente del consumo más intensivo y mayor de los jóvenes. Aunque esta explicación no se apoya totalmente con los datos de la encuesta nacional ESTUDES, que muestra que los adolescentes consideran un riesgo similar el consumir 5 o 6 bebidas alcohólicas los fines de semana y tomar 2 bebidas alcohólicas diariamente (Ministerio de Sanidad, 2018a). Ambas circunstancias pueden implicar una infraestimación del consumo de alcohol de los progenitores.

La mayor proporción de expectativas positivas respecto al consumo de alcohol entre aquellos sujetos con mayores percepciones del consumo de sus madres, padres, hermanos/as, amigos/as e incluso del consumo propio, refleja un paralelismo entre estas variables, reforzando la relación previamente expuesta en la introducción del manuscrito. Consecuentemente la direccionalidad que aplicamos a esta relación nos lleva a entender las expectativas -que se han mostrado influyentes en el CRA y CIA tanto en la Cohorte Compostela 2005 (Moure-Rodríguez et al., 2018), como en otros grupos de jóvenes (Anderson, Grunwald, Bekman, Brown y Grant, 2011; Wicki et al., 2010)- como una variable intermedia en el modelo.

La tendencia temporal de estas prácticas de consumo en gente joven es por lo general en forma de campana, alcanzando un pico máximo para luego comenzar a disminuir (Andersson, Johnsson, Berglund, y Ojehagen, 2007; Bewick et al., 2008). Los resultados presentados en este estudio muestran que, quienes consideran el consumo de alcohol propio y de los amigos/as como escaso o ninguno, 
Tabla 5. Porcentajes de hombres que practican consumo de riesgo y consumo intensivo de alcohol entre los 18 y los 27 años en función de su percepción de consumo propio y de sus allegados a los 18-19.

\begin{tabular}{|c|c|c|c|c|c|c|c|c|c|c|}
\hline & \multicolumn{5}{|c|}{ Consumo de Riesgo de Alcohol (\%) } & \multicolumn{5}{|c|}{ Consumo Intensivo de Alcohol (\%) } \\
\hline & \multicolumn{5}{|c|}{ Edad } & \multicolumn{5}{|c|}{ Edad } \\
\hline & 18 & 20 & 22 & 24 & 27 & 18 & 20 & 22 & 24 & 27 \\
\hline \multicolumn{11}{|c|}{ Percepción de consumo propio } \\
\hline No consumo & 3,3 & 15,4 & 14,3 & 6,2 & 0 & 1,7 & 5,1 & 21,4 & 0,0 & , o \\
\hline Consumo poco & 37,7 & 56,6 & 53,4 & 13,5 & 39,3 & 12,3 & 24,1 & 34,5 & 8,1 & 7,9 \\
\hline Consumo bastante & 96,0 & 90,5 & 78,0 & 36,4 & 46,4 & 66,4 & 68,3 & 58,5 & 27,3 & 40,7 \\
\hline Consumo mucho & $100^{\star}$ & $100^{\star}$ & $83,3^{*}$ & $66,7^{\star}$ & $14,3^{*}$ & $85,7^{\star}$ & $78,9^{\star}$ & $83,3^{\star}$ & $83,3^{\star}$ & $44,4^{\star}$ \\
\hline \multicolumn{11}{|c|}{ Percepción del consumo de alcohol de los amigos/as } \\
\hline No consumen & 20,0 & - & - & - & & 0,0 & 0,0 & - & - & - \\
\hline Consumen poco & 23,3 & 31,0 & 35,5 & 22,2 & 6,2 & 9,5 & 19,7 & 2,8 & 16,7 & 6,2 \\
\hline Consumen bastante & 58,8 & 68,4 & 58,1 & 20,5 & 37,2 & 32,4 & 37,0 & 41,9 & 15,4 & 23,3 \\
\hline Consumen mucho & $80,7^{\star}$ & $77,4^{*}$ & $67,4^{*}$ & 26,1 & $37,9^{*}$ & $58,8^{\star}$ & $58,1^{\star}$ & $58,1^{\star}$ & 21,7 & 24,1 \\
\hline \multicolumn{11}{|c|}{ Percepción del consumo de alcohol de hermanos/as } \\
\hline No consumen & 54,6 & 58,7 & 54,9 & 29,3 & 31,9 & 32,4 & 33,7 & 42,3 & 24,4 & 21,3 \\
\hline Consumen poco & 57,8 & 59,1 & 54,8 & 18,2 & 15,5 & 41,0 & 36,4 & 45,3 & 13,6 & 20,0 \\
\hline Consumen bastante & 64,6 & 66,7 & 56,2 & 12,5 & 33,3 & 39,6 & 54,2 & 37,5 & 12,5 & 22,2 \\
\hline Consumen mucho & $100^{\star}$ & $100^{*}$ & 80,0 & - & - & $71,4^{\star}$ & $70,0^{\star}$ & 80,0 & - & 25,0 \\
\hline \multicolumn{11}{|c|}{ Percepción del consumo de alcohol de la madre } \\
\hline No consume & 56,9 & 63,7 & 52,5 & 22,2 & 30,8 & 33,8 & 45,2 & 41,2 & 14,0 & 19,2 \\
\hline Consume poco & 57,1 & 58,8 & 56,4 & 25,0 & 28,6 & 37,0 & 34,1 & 41,8 & 25,0 & 20,0 \\
\hline Consume bastante & 100 & - & - & - & & 80,0 & 33,3 & - & - & - \\
\hline Consume mucho & & & & & & - & - & - & - & - \\
\hline \multicolumn{11}{|c|}{ Percepción del consumo de alcohol del padre } \\
\hline No consume & 54,8 & 65,5 & 56,8 & 34,8 & 33,3 & 32,7 & 44,2 & 43,2 & 26,1 & 29,2 \\
\hline Consume poco & 56,5 & 60,6 & 52,4 & 17,0 & 26,0 & 35,9 & 39,4 & 42,7 & 12,8 & 14,0 \\
\hline Consume bastante & 80,0 & 68,4 & 64,3 & 33,3 & 50,0 & 43,3 & 26,3 & 35,7 & 33,3 & 40,0 \\
\hline Consume mucho & 66,7 & 66,7 & - & - & - & 66,7 & 33,3 & - & - & - \\
\hline Total de sujetos & 58,0 & 62,6 & 55,4 & 22,2 & $31,1^{\wedge}$ & 35,6 & 38,8 & 43,2 & 17,3 & $20,0^{\wedge}$ \\
\hline
\end{tabular}

Nota.

* Diferencias significativas entre categorías de percepción de consumo. $X^{2}, p<0.05$

$\wedge$ Diferencias significativas entre edades. Test $X^{2}$, p<0.05

alcanzan el pico máximo considerablemente más tarde, a los 22 años, y aun así no llegan a alcanzar las prevalencias de CRA de sus pares. Esto nos indica como probablemente una parte de este subgrupo de jóvenes comenzaron estas prácticas de consumo durante su periodo universitario. Comenzar estas prácticas en la universidad es un fenómeno que ha sido observado por otros autores previamente (Weitzman, Nelson, y Wechsler, 2003), y que señala la importancia de tomar medidas preventivas durante este periodo vital. Por otra parte, refuerza el potencial efecto protector del consumo de los amigos más allá del primer año de universidad.

Pasando al análisis multivariante, la asociación más intensa se ha establecido entre la percepción de consumo de alcohol de los amigos y la práctica de CRA y CIA de las mujeres y la práctica de CIA entre los hombres, lo que va en línea con la literatura, donde el consumo de alcohol de los universitarios, o incluso su práctica de CIA puede predecirse por el consumo de alcohol de sus pares (Borsari, Murphy y Barnett, 2007; Dumas, Davis, Maxwell-Smith y Bell, 2018; Robinson, Jones, Christiansen y Field, 2015). En este estudio hemos preguntado a los participantes por el consumo de alcohol de sus amigos, por tanto, estamos evaluando el efecto específico del comportamiento de sus más allegados. Es lógico pensar que los amigos tengan un efecto más importante en los universitarios que sus pares en general, pues son las personas con las que más tiempo pasan. Esto resulta coincidente con la literatura científica, donde se ha encontrado que los amigos cercanos o grupos referentes más similares a uno han mostrado mayor influencia en el consumo de alcohol de los jóvenes (Larimer et al., 2009; Mallett, Bachrach y Turrisi, 2009). En 


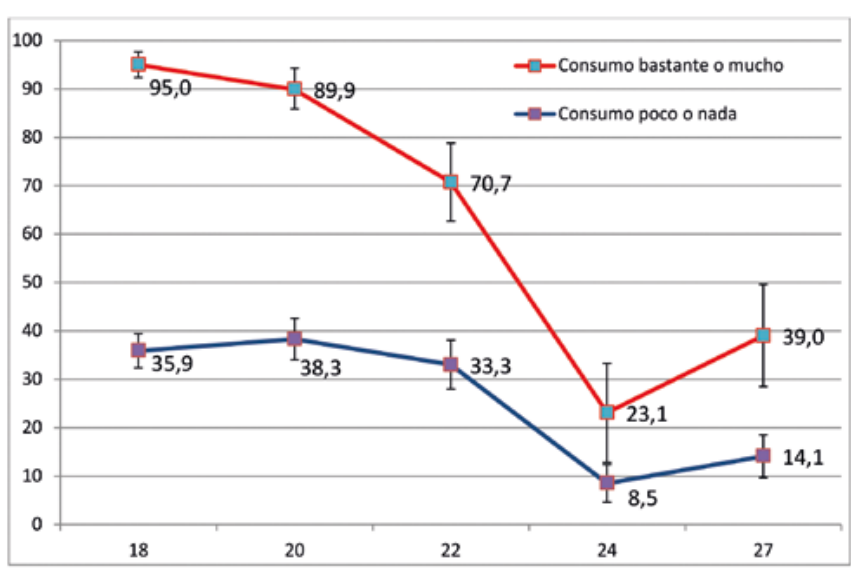

Figura 1. Prevalencia (\%) de consumo de riesgo de alcohol entre las mujeres a los 18, 20, 22, 24 y 27 años en función de la percepción del propio consumo a los 18 años.

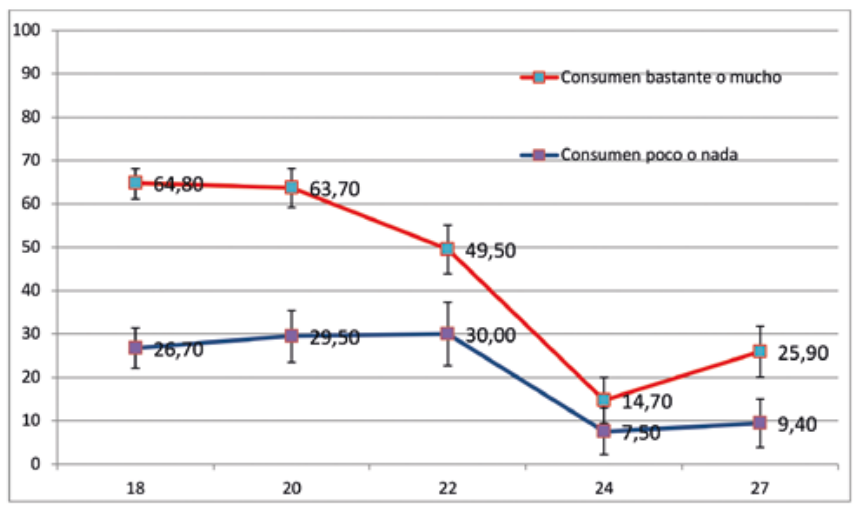

Figura 3. Prevalencia (\%) de consumo de riesgo de alcohol entre las mujeres a los 18, 20, 22, 24 y 27 años en función de la percepción de consumo de sus amigos a los 18.

esta línea Larimer et al. (2009) concluye que realizar un feedback sobre el consumo de grupos de referencia con más afinidad será más efectivo. Por otra parte, Mallet et al. (2009) estudian la percepción de consumo de los universitarios respecto a grupos de referencia, estudiantes del mismo sexo y amigos, resultado este último grupo el único influyente en el consumo de los jóvenes participantes. En estos resultados observamos como la influencia de una percepción de mayor consumo de alcohol de los amigos a los 18 años aumenta el riesgo de practicar ambos patrones de consumo a lo largo de 9 años se seguimiento, más allá del periodo universitario. Esto puede verse también en las Figuras 3 y 4, que muestran una clara diferencia en las prevalencias de CRA en función de la percepción del consumo de los amigos/as. Incluso a pesar del aumento de las prevalencias de CRA hasta los 22 años entre quienes consideraban a los 18 años que sus amigos consumían poco o nada, las prevalencias se mantienen por debajo de las de sus pares durante todo el periodo de seguimiento, $\mathrm{y}$ solo se solapan durante la caída más importante de CRA, a los 24 años.

Estos resultados subrayan la importancia de actuar sobre la percepción de consumo de los amigos/as en progra-

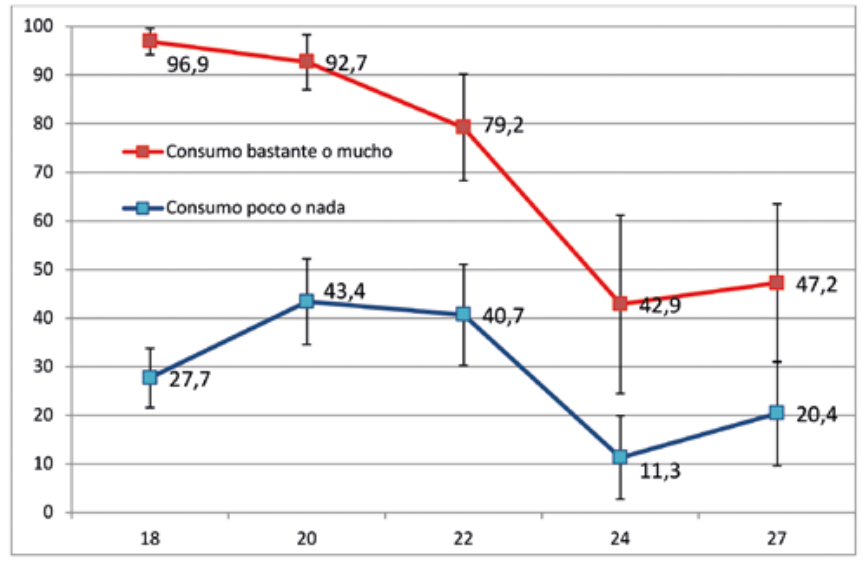

Figura 2. Prevalencia (\%) de consumo de riesgo de alcohol entre los hombres a los 18, 20, 22, 24 y 27 años en función de la percepción del propio consumo a los 18 años.

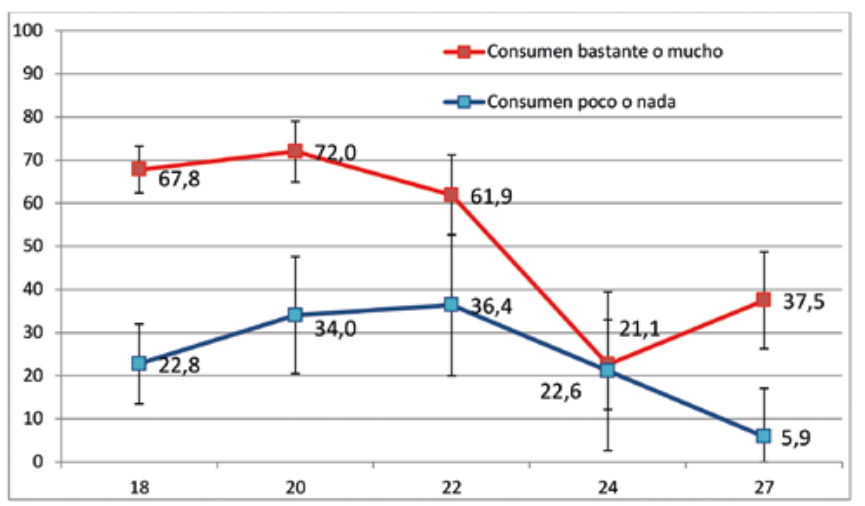

Figura 4. Prevalencia (\%) de consumo de riesgo de alcohol entre los hombres a los 18, 20, 22, 24 y 27 años en función de la percepción de consumo de sus amigos a los 18.

mas de prevención de consumo durante la adolescencia y la juventud, no solo para prevenir los consumos de riesgo el primer año de universidad, si no durante los años posteriores. En este sentido, la publicidad dirigida específicamente a los jóvenes (con fotografías de gente de su edad bebiendo alcohol) es especialmente perjudicial. La regulación de la publicidad dirigida a los jóvenes es un paso esencial para evitar la normalización del consumo de alcohol a estas edades (Sargent y Babor, 2020).

Aunque la literatura no es homogénea a este respecto, algunos autores han encontrado evidencia que sugiere que las mujeres tienden a verse más influenciadas por el consumo de alcohol de sus amigos/as. En esta línea Simons-Morton et al. (1999) refieren que tener amigos problemáticos aumenta el riesgo de consumo de alcohol solo entre las mujeres adolescentes, mientras Gaughan (2006) encuentra influencias del consumo de amigos del género opuesto solo entre las mujeres. Esto puede explicar en parte la falta de efecto de esta variable en el análisis multivariante para el CRA en los hombres.

Podría argumentarse que una posible limitación del estudio es la falta de información respecto al consumo real de los allegados, pues tomamos esta información directa- 
Tabla 6. Influencia del propio consumo y del consumo de los allegados a los 18 años en el consumo de riesgo y consumo intensivo de alcohol de las universitarias de los 18 a los 27 años.

\begin{tabular}{|c|c|c|c|c|}
\hline & \multicolumn{4}{|c|}{ Odds ratio $(95 \% \mathrm{Cl})$} \\
\hline & \multicolumn{2}{|c|}{ Consumo de riesgo de alcohol } & \multicolumn{2}{|c|}{ Consumo Intensivo de Alcohol } \\
\hline & Bivariante & Multivariante $^{\mathrm{a}}$ & Bivariante & Multivariante $^{a}$ \\
\hline \multicolumn{5}{|c|}{ Percepción del consumo de alcohol de los amigos/as } \\
\hline No consumen & 1 & 1 & 1 & 1 \\
\hline Consumen poco & $5,7(2,0-16,3)$ & $3,8(1,3-11,4)$ & $3,7(0,4-31,1)$ & $2,2(0,3-17,7)$ \\
\hline Consumen bastante & $18,1(6,4-51,4)$ & $11,2(3,8-32,7)$ & $13,0(1,6-107,4)$ & $6,9(0,9-55,7)$ \\
\hline Consumen mucho & $30,4(10,6-87,4)$ & $17,5(5,8-52,2)$ & $34,6(4,2-288,2)$ & $19,3(2,4-156,6)$ \\
\hline \multicolumn{5}{|c|}{ Percepción del consumo de alcohol de hermanos/as } \\
\hline No consumen & 1 & 1 & 1 & 1 \\
\hline Consumen poco & $1,2(1,0-1,5)$ & $1,3(1,3-1,6)$ & $1,4(1,0-1,8)$ & $1,1(0,9-1,4)$ \\
\hline Consumen bastante & $3,4(2,6-4,5)$ & $2,9(2,2-3,8)$ & $2,6(1,9-3,6)$ & $2,7(2,0-3,6)$ \\
\hline Consumen mucho & $5,0(2,9-8,8)$ & $3,7(2,0-7,0)$ & $5,7(3,3-9,7)$ & $4,5(2,4-8,4)$ \\
\hline \multicolumn{5}{|c|}{ Percepción del consumo de alcohol de la madre } \\
\hline No consume & 1 & & 1 & \\
\hline Consume poco & $1,0(0,8-1,1)$ & & $1,1(0,9-1,4)$ & \\
\hline Consume bastante & $2,5(0,9-6,8)$ & & $0,7(0,2-3,2)$ & \\
\hline \multicolumn{5}{|c|}{ Percepción del consumo de alcohol del padre } \\
\hline No consume & 1 & & 1 & \\
\hline Consume poco & $1,4(1,1-1,6)$ & & $1,1(0,9-1,4)$ & \\
\hline Consume bastante & $1,9(1,3-2,6)$ & & $1,4(0,9-2,2)$ & \\
\hline Consume mucho & $0,1(0,03-0,6)$ & & $0,3(0,04-2,4)$ & \\
\hline \multicolumn{5}{|l|}{ Lugar de residencia } \\
\hline Domicilio familiar & 1 & 1 & 1 & 1 \\
\hline Fuera del domicilio familiar & $1,6(1,3-2,0)$ & $1,9(1,5-2,4)$ & $1,6(1,2-2,1)$ & $1,7(1,2-2,3)$ \\
\hline
\end{tabular}

Nota.

${ }^{a}$ Ajustada por el resto de las variables incluidas en la columna y el nivel de educación materna y la edad de los participantes (periodo).

mente de los participantes, pero estudios previos que han puesto el foco en adolescentes y jóvenes han encontrado que las percepciones que tienen del consumo de alcohol de sus pares son un mejor predictor de su consumo que el consumo real que sus pares practican (Deutsch, Chernyavskiy, Steinley y Slutske, 2015; Kenney, Ott, Meisel y Barnett, 2017). Además, la elevada prevalencia de CRA y CIA encontrada en la cohorte a los 18 y 19 años $(58,0 \%$ y $35,6 \%$ en hombres y $51,5 \%$ y $17,9 \%$ en mujeres respectivamente) apoya la correcta percepción de consumo de los amigos referida por los participantes.

Nuestros resultados muestran que la percepción de que los hermanos o hermanas consumen mucho alcohol aumenta el riesgo de CRA y CIA en ambos géneros. Aunque la evidencia en la literatura a este respecto es escasa, los resultados alcanzados van en línea con estudios previos que muestran el consumo de los hermanos mayores como un importante predictor del consumo entre los jóvenes (Stormshak, Comeau y Shepard, 2004; Whiteman, Jensen y Maggs, 2013). Los hermanos forman parte del contexto familiar en un periodo vital en que la influencia de los padres parece disminuir a medida que los jóvenes se interrelacionan entre sí y se ven más influidos por sus pares (Aseltine, 1995; Voogt et al., 2017). Probablemente los hermanos y/o hermanas tienen una relación entre sí más similar a las de los pares (Schuler, Tucker, Pedersen y D’Amico, 2019; Serafini y Stewart, 2015), al estar más cerca en edad, es posible que se sientan más en harmonía entre sí, compartan más intereses y momentos vitales similares. Los hermanos o hermanas mayores pueden entenderse como modelos de conducta, y su consumo de alcohol, por tanto, influir a los menores. En un estudio a este respecto (Whiteman,Jensen, Mustillo y Maggs, 2016), tras tener en cuenta los amigos compartidos entre hermanos, Whiteman et al., se muestran a favor de la hipótesis planteada por numerosos autores en población escolar sobre la influencia de los hermanos mayores en el consumo de los menores, probablemente facilitando el acceso al alcohol e incluso a lugares donde consumirlo.

No se ha encontrado asociación de la percepción de consumo de alcohol de los padres o madres con el patrón de CIA o CRA. Esta falta de asociación puede deberse en 
Tabla 7. Influencia del propio consumo y del consumo de los allegados a los 18 años en el consumo de riesgo y consumo intensivo de alcohol de los universitarios entre los 18 y los 27 años.

\begin{tabular}{|c|c|c|c|c|}
\hline & \multicolumn{4}{|c|}{ Odds ratio (IC 95\%) } \\
\hline & \multicolumn{2}{|c|}{ Consumo de Riesgo de Alcohol } & \multicolumn{2}{|c|}{ Consumo Intensivo de alcohol } \\
\hline & Bivariante & Multivariante $^{a}$ & Bivariante & Multivariante $^{a}$ \\
\hline \multicolumn{5}{|c|}{ Percepción del consumo de alcohol de los amigos/as } \\
\hline No consumen & 1 & & 1 & 1 \\
\hline Consumen poco & $0,6(0,2-1,9)$ & ¿??? & $2,8(0,3-25,9)$ & $2,7(0,3-27,4)$ \\
\hline Consumen bastante & $2,1(0,6-6,8)$ & & $7,2(0,8-63,2)$ & $7,0(0,7-67,3)$ \\
\hline Consumen mucho & $4,1(1,3-13,4)$ & & $17,9(2,0-158,8)$ & $17,5(1,8-170,3)$ \\
\hline \multicolumn{5}{|c|}{ Percepción del consumo de alcohol de hermanos/as } \\
\hline No consumen & 1 & 1 & 1 & 1 \\
\hline Consumen poco & $0,9(0,6-1,3)$ & $0,8(0,6-1,2)$ & $1,1(0,8-1,6)$ & $1,1(0,7-1,6)$ \\
\hline Consumen bastante & $1,3(0,8-2,1)$ & $1,2(0,7-1,9)$ & $1,3(0,8-2,1)$ & $1,2(0,7-2,0)$ \\
\hline Consumen mucho & $11,7(3,3-41,9)$ & $11,6(3,3-40,9)$ & $4,2(2,0-9,1)$ & $2,8(1,2-6,6)$ \\
\hline \multicolumn{5}{|c|}{ Percepción del consumo de alcohol de la madre } \\
\hline No consume & 1 & & 1 & \\
\hline Consume poco & $1,0(0,7-1,3)$ & & $1,3(0,7-1,4)$ & \\
\hline Consume bastante & $8,5(1,6-46,6)$ & & $1,8(0,7-5,1)$ & \\
\hline \multicolumn{5}{|c|}{ Percepción del consumo de alcohol del padre } \\
\hline No consume & 1 & & 1 & \\
\hline Consume poco & $0,9(0,6-1,2)$ & & $0,8(0,6-1,2)$ & \\
\hline Consume bastante & $1,7(1,0-3,1)$ & & $0,9(0,5-1,6)$ & \\
\hline Consume mucho & $0,8(0,3-2,4)$ & & $0,8(0,3-2,4)$ & \\
\hline \multicolumn{5}{|l|}{ Lugar de residencia } \\
\hline Domicilio familiar & 1 & 1 & 1 & 1 \\
\hline Fuera del domicilio familiar & $1,6(1,2-2,2)$ & $1,6(1,1-2,3)$ & $1,6(1,1-2,2)$ & $1,6(1,1-2,4)$ \\
\hline
\end{tabular}

Nota.

${ }^{a}$ Ajustada por el resto de las variables incluidas en la columna y el nivel de educación materna y la edad de los participantes (periodo).

parte a la escasa variabilidad de estas variables. Nuestros resultados muestran que la percepción de que los padres consumen poco o bastante alcohol aumenta el riesgo de mostrar CRA entre las mujeres y la percepción de que las madres consumen bastante alcohol aumenta el riesgo de CRA entre los hombres, en ambos casos en el análisis bivariante. En esta línea se ha encontrado una asociación en el análisis bivariante del consumo de alcohol de padres y madres y el consumo de los universitarios en 3.418 jóvenes de 7 universidades del estado de Georgia (Windle, Haardörfer, Lloyd, Foster y Berg, 2017).

Sin embargo, que el padre consuma mucho alcohol actúa como un factor protector entre las mujeres. Una posible explicación es que ver consumir alcohol con regularidad al padre normalice el consumo, pero solo hasta un determinado punto en el que los efectos negativos de este consumo se hacen más evidentes. Este fenómeno se ha descrito previamente en la literatura y se conoce como "transmisión aversiva", y consiste precisamente en que a medida que los padres consumen más alcohol lo hacen los descendientes, pero cuando esas cantidades son muy ele- vadas, los niveles disminuyen considerablemente entre los descendientes, y específicamente entre las hijas (Harburg, DiFranceisto, Webster, Gleiberman y Schork, 1990). Esta asociación desaparece cuando otras variables entran en juego, como el consumo de alcohol de los amigos. Windle et al. (2017) explican esto como el reflejo de la menor influencia de los padres y madres a medida que los hijos e hijas crecen, lo que va en línea con el efecto más intenso que tendrá en el consumo de sustancias entre los jóvenes las actitudes y consumos de personas y grupos con los que interactúan más frecuentemente y de un modo más intenso (Bahr et al., 2005).

Merece una atención especial el hecho de que las asociaciones entre la percepción de consumo de los padres se establecen con el CRA en el análisis bivariante, mientras que la asociación de las percepciones de consumo de alcohol de los amigos se asocia al CIA en ambos géneros, un patrón más habitual entre la gente joven (Ministerio de Sanidad, 2018b).

Vivir fuera del domicilio familiar aumenta el riesgo de CRA y CIA en universitarios, una variable claramente re- 
ferida al contexto familiar, y que mantiene su efecto en el análisis multivariante. Esto significa que a la misma percepción de consumo de alcohol de los amigos, padre, madre o hermanos/as, los universitarios que viven en el domicilio familiar presentan un menor riesgo de CRA y CIA. Esta variable ha presentado influencia previamente sobre estas prácticas de consumo en la Cohorte Compostela 2005 (Moure-Rodríguez et al., 2016), y la entendemos como un reflejo de la mayor supervisión o monitorización parental de los jóvenes que viven en el domicilio familiar, observada así mismo por otros autores, como un factor protector del consumo de alcohol en gente joven (Moore, Rothwell y Segrot t, 2010). Algunos autores han referido que la mayor monitorización parental entendida como conocimiento de con quien se relacionan sus hijos e hijas o lo que hacen, o simplemente la mayor sensación de participación por parte de los estudiantes, actúa como un factor protector del consumo de alcohol, incluso durante el periodo universitario (Abar y Turrisi, 2008; Jessor, Costa, Krueger y Turbin, 2006; Wood et al., 2004). Por tanto, vivir con los padres durante el periodo universitario puede disminuir la influencia de los pares respecto al consumo de alcohol (White, Fleming, Kim, Catalano y Mcmorris, 2008). Quinn et al. (2011) llevaron a cabo un estudio en que compararon a jóvenes universitarios y jóvenes no universitarios, llegando a la conclusión de que las normas sociales de grupo afectan menos a los no universitarios, probablemente porque los primeros tienden a vivir con sus pares, y por tanto están más influenciados por ellos. Esta relación cerrada y continua con sus pares no será tan intensa con jóvenes que viven en el domicilio familiar. Además, la presión de los pares en el campus favorecerá la promoción del CIA, actuando los pares como proveedores de alcohol, los mayores pueden actuar como modelos de conducta y el entorno ayuda por tanto a que la práctica de CIA sea habitual y aceptada (Borsari y Carey, 2001).

A la vista de estos resultados, las medidas preventivas deberían diseñarse en el futuro desde una perspectiva contextual, en donde no solo los jóvenes universitarios, sino también sus allegados -amigos/as, hermanos/as- se tengan en cuenta. En esta línea Lewis y Neighbors (2006) concluyen, en una revisión de la literatura científica, que un feedback personalizado del consumo de los pares ha sido efectivo disminuyendo el consumo de alcohol y sus consecuencias negativas entre los jóvenes. Hemos confirmado la importancia de la influencia de las relaciones sociales en estas prácticas entre nuestros universitarios. Además, el hecho de vivir en el domicilio familiar al inicio del periodo universitario continúa actuando como factor protector, no solo durante los primeros años de universidad, sino durante los siguientes 10 años, lo que señala la influencia del contexto familiar, que sigue protegiendo de estas condutas de riesgo incluso en una sociedad donde el consumo de alcohol está muy normalizado (Ministerio de Sanidad, Política Social e Igualdad, s.f.).
Este estudio cuenta con cuatro limitaciones principales: 1) como en otros estudios de cohortes, la pérdida de sujetos durante el seguimiento puede llevar a sesgos. Sin embargo, no ha habido diferencias significativas entre los participantes a lo largo del periodo de estudio, sugiriendo la ausencia de estos sesgos; 2) Sesgo de información, siempre probable en cuestionarios autocumplimentados. Para minimizar este sesgo se ha utilizado el AUDIT, un cuestionario validado internacionalmente entre adolescentes y adultos jóvenes; 3) La tercera pregunta del AUDIT no permite diferenciar entre géneros, por tanto, la prevalencia del CIA en mujeres puede estar infraestimada en este estudio, al no tener en cuenta las mujeres que consumen 5 bebidas en una única ocasión. Aun así, esto solo afecta al análisis descriptivo y no a los resultados analíticos, y 4) el cuestionario sobre expectativas no está validado y por tanto es posible que las expectativas respecto al consumo de alcohol no hayan sido medidas correctamente.

\section{Conclusión}

Los universitarios perciben mucho más consumo de alcohol entre sus amigos y mucho más consumo propio que entre sus familiares. A medida que la percepción de consumo de alcohol de los allegados aumenta lo hace la proporción de sujetos que practican CRA y CIA. El riesgo de practicar CIA o CRA aumenta en ambos géneros cuando los universitarios perciben que sus hermanos/as consumen grandes cantidades de alcohol. Esta asociación también es cierta respecto al consumo de sus amigos, excepto por la ausencia de la misma para el CRA en los hombres. Los patrones de consumo de alcohol de los padres no afectan al consumo de los universitarios cuando los amigos/as y hermanos/as se consideran. Finalmente, vivir fuera del domicilio familiar aumenta el riesgo de ambos patrones de consumo entre universitarias y universitarios. En vista a estos resultados las medidas preventivas deben diseñarse en el futuro teniendo en cuenta el contexto y los allegados, y, no solo a los jóvenes diana.

\section{Reconocimientos}

Este trabajo ha sido financiado por el Plan Nacional sobre Drogas (Spain) (2005/PN014) y el Fondo de Investigación Sanitaria (Spain) (PI15/00165). Carina Carbia ha sido financiada por el European Union's Horizon 2020 research and innovation programme con la beca Marie Sklodowska-Curie No. 754535.

\section{Conflicto de Intereses}

Los autores no tienen conflicto de intereses que declarar. 


\section{Referencias}

Abar, C. C. y Turrisi, R. (2008). How important are parents during the college years? A longitudinal perspective of indirect influences parents yield on their college teens' alcohol use. Addictive Behaviors, 33,1360-1368. doi:10.1016/j.addbeh.2008.06.010.

Anderson, K. G., Grunwald, I., Bekman, N., Brown, S.A. y Grant, A. (2011). To drink or not to drink: motives and expectancies for use and nonuse in adolescence. Addictive Behaviors, 36, 972-979. doi:10.1016/j.addbeh.2011.05.009.

Andersson, C., Johnsson, K.O., Berglund, M. y Ojehagen, A. (2007). Alcohol involvement in Swedish university fresh men related to gender, age, serious relations hip and family history of alcohol problems. Alcohol and Alcoholism, 42, 448-455. doi:10.1093/alcalc/agm008.

Aseltine, R. H. (1995). A reconsideration of parental and peer influences on adolescent deviance. Journal of Health and Social Behavior, 36, 103-121.

Bahr, S. J., Hoffmann, J. y Yang, X. (2005). Parental and peer influences on the risk of adolescent drug use. The Journal of Primary Prevention, 26, 529-551. doi:10.1007/ s10935-005-0014-8.

Bava, S. y Tapert, S. F. (2010). Adolescent brain development and the risk for alcohol and other drug problems. Neuropsychology review, 20, 398-413. doi:10.1007/s11065010-9146-

Benz, M. B., DiBello, A. M., Balestrieri, S. G., Miller, M. B., Merrill, J.E., Lowery, A.D., ...Carey, K. B. (2017). Off-campus residence as a risk factor for heavy drinking among college students. Substance Use and Misuse, 52, 1133-1138. doi:10.1080/10826084.2017.1298620.

Bewick, B. M., Mulhern, B., Barkham, M., Trusler, K., Hill, A. J. y Stiles, W. B. (2008). Changes in undergraduate student alcohol consumption as they progress through university. BMC Public Health, 8,163. doi:10.1186/14712458-8-163.

Borsari, B. y Carey, K. B. (2001). Peer influences on college drinking: A review of the research. Journal of Substance Abuse, 13, 391-424.

Borsari, B., Murphy, J. G. y Barnett, N. P. (2007). Predictors of alcohol use during the first year of college: Implications for prevention. Addictive Behaviors, 32, 2062-2086. doi:10.1016/j.addbeh.2007.01.017.

Brown, B. B. y Larson, J. (2009). Peer relationships in adolescence. En R.M. Lerner y L. Steinberg (Eds.), Handbook of Adolescent Psychology (pp. 74-103). New York: Wiley y Sons.

Center for Behavioral Health Statistics and Quality. (2015). 2014 National Survey on Drug Use and Health Detailed Tables (NSDUH). Rockville, MD: Substance Abuse and Mental Health Services Administration (SAMHSA).

Chassin, L., Pitts, S. C. y Prost, J. (2002). Binge drinking trajectories from adolescence to emerging adulthood in a high-risk sample: predictors and substance abuse outcomes. Journal of Consulting and Clinical Psychology, 70, 67-78.

Cox, M. J., DiBello, A. M., Meisel, M. K., Ott, M. Q., Kenney, S. R., Clark, M. A. y Barnett, N. P. (2019). Do misperceptions of peer drinking influence personal drinking behavior? Results from a complete social network of first-year college students. Psychology of Addictive Behaviors, 33, 297-303. doi:10.1037/adb0000455.

Critchlow, B. (1986). The powers of John Barleycorn: beliefs about the effects of alcohol on social behavior. The American Psychologist, 41, 751-764.

Crundall, I. A. (1995). Perceptions of alcohol by student drinkers at university. Drug and Alcohol Review, 14, 363368.

Cservenka, A. y Brumback, T. (2017). The burden of binge and heavy drinking on the brain: effects on adolescent and young adult neural structure and function. Frontiers in Psychology, 8,1111. doi:10.3389/fpsyg.

Defensor del Menor de la Comunidad de Madrid. (2003). Análisis del consumo de alcohol por los jóvenes en la Comunidad de Madrid. En El Defensor del Menor de la Comunidad de Madrid (Eds.), Defensor del Menor de la Comunidad de Madrid: Estudios e investigaciones 2002 (pp. 307-390). Madrid: Defensor del Menor en la Comunidad de Madrid.

Deutsch, A. R., Chernyavskiy, P., Steinley, D. y Slutske, W. S. (2015). Measuring peer socialization for adolescent substance use: A comparison of perceived and actual friends' substance use effects. Journal of Studies on Alcohol and Drugs, 76, 267-277. doi:10.15288/jsad.2015.76.267.

Dumas, T. M., Davis, J. P., Maxwell-Smith, M. A. y Bell, A. (2018). From drinking group norms to individual drinking consequences: A moderated mediation model examining the role of members' status, identification with the group and with emerging adulthood. Substance Use and Misuse, 53, 1311-1323. doi:10.1080/10826084.2017 .1408651 .

Dumas, T. M., Davis, J. P. y Neighbors, C. (2019). How much does your peer group really drink? Examining the relative impact of overestimation, actual group drinking and perceived campus norms on university students' heavy alcohol use. Addictive Behaviors, 90, 409-414. doi:10.1016/j.addbeh.2018.11.041.

ESPAD Group. (2016). ESPAD Report 2015: Results from the European School Survey Project on Alcohol and Other Drugs. Luxembourg: Publications Office of the European Union.

Farke, W. y Anderson, P. (2007). Binge drinking in Europe. Adicciones, 19, 333-339.

Galán, I., González, M. J. y Valencia-Martín, J. L. (2014). Patrones de consumo de alcohol en España: un país en transición. Revista Española de Salud Publica, 88, 529-540. doi: 10.4321/S1135-57272014000400007. 
Gaughan, M. (2006). The gender structure of adolescent peer influence on drinking. Journal of Health and Social Behavior, 47, 47-61. doi:10.1177/002214650604700104.

Gual, A., Ángel Arbesú, J., Zarco, J., Balcells-Oliveró, M.L.M., López-Pelayo, H., Miquel, L. y Bobes, J. (2017). Risky drinkers underestimate their own alcohol consumption. Alcohol and Alcoholism, 52, 516-517. doi:10.1093/alcalc/agx029.

Harburg, E., DiFranceisto, W., Webster, D.W., Gleiberman, L. y Schork, A. (1990). Familial transmission of alcohol use: II. Imitation of and aversion to parent drinking (1960) by adult offspring (1977)-Tecumseh, Michigan. Journal of Studies on Alcohol, 51, 245-256. doi:10.15288/ jsa.1990.51.245.

Jefferis, B. J., Power, C. y Manor, O. (2005). Adolescent drinking level and adult binge drinking in a national birth cohort. Addiction, 100, 543-549. doi:10.1111/ j.1360-0443.2005.01034.x.

Jessor, R., Costa, F. M., Krueger, P. M. y Turbin, M. S. (2006). A developmental study of heavy episodic drinking among college students: the role of psychosocial and behavioral protective and risk factors. Journal of Studies on Alcohol, 67, 86-94.

Kenney, S. R., Ott, M., Meisel, M. K. y Barnett, N. P. (2017). Alcohol perceptions and behavior in a residential peer social network. Addictive Behaviors, 64, 143-147. doi:10.1016/j.addbeh.2016.08.047.

Kuntsche, E., Rehm, Jy Gmel, G. (2004). Characteristics of binge drinkers in Europe. Social Science $\mathcal{E}$ Medicine, 59, 113-127. doi:10.1016/j.socscimed.2003.10.009

Larimer, M. E., Kaysen, D. L., Lee, C. M., Kilmer, J. R., Lewis, M. A., Dillworth, T., ... Neighbors, C. (2009). Evaluating level of specificity of normative referents in relation to personal drinking behavior. Journal of Studies on Alcohol and Drugs, 16, 115-121. doi:10.15288/jsads.2009.s16.115.

Lewis, M. A. y Neighbors, C. (2006). Social norms approaches using descriptive drinking norms education: A review of the research on personalized normative feedback. Journal of American College Health, 54, 213-218.

Mallett, K. A., Bachrach, R. L. y Turrisi, R. (2009). Examining the unique influence of interpersonal and intrapersonal drinking perceptions on alcohol consumption among college students. Journal of Studies on Alcohol and Drugs, 70, 178-185. doi:10.15288/jsad.2009.70.178.

Martinotti, G., Lupi, M., Carlucci, L., Santacroce, R., Cinosi, E., Acciavatti, T., ... Di Giannantonio, M. (2017). Alcohol drinking patterns in young people: A survey-based study. Journal of Health Psychology, 22, 1889-1896. doi:10.1177/1359105316667795.

Meier, P. S. (2010). Polarized Drinking Patterns and Alcohol Deregulation: Trends in alcohol consumption, harms and policy: United Kingdom 19902010. Nordic Studies on Alcohol and Drugs, 27, 383-408. doi:10.1177/145507251002700501.
Merrill, J. E. y Carey, K. B. (2016). Drinking over the lifespan: Focus on college ages. Alcohol Research, 38, 103-114.

Miller, P. M., Smith, G. T. y Goldman, M.S. (1990). Emergence of alcohol expectancies in childhood: a possible critical period. Journal of Studies on Alcohol, 51, 343-349.

Ministerio de Sanidad, Política Social e Igualdad. Delegación del Gobierno para el Plan Nacional sobre Drogas. (2018a). Informe 2018. Alcohol, tabaco y drogas ilegales en España. Encuesta sobre Uso de Drogas en Enseñanzas Secundarias en España (ESTUDES) 1994-2016. Madrid: Ministerio de Sanidad, Política Social e Igualdad.

Ministerio de Sanidad, Política Social e Igualdad. Delegación del Gobierno para el Plan Nacional sobre Drogas. (2018b). Encuesta Sobre Alcohol y otras drogas en España (EDADES) 1995-2017. Madrid: Ministerio de Sanidad, Política Social e Igualdad.

Ministerio de Sanidad, Política Social e Igualdad. (s.f.). Situación actual del consumo de alcohol en España. Ministerio de Sanidad, Política Social e Igualdad. Recuperado de http:/ / www.mscbs.gob.es/campannas/campanas10/ embarazadasCeroAlcohol/situacion_actual_del_consumo_de_alcohol.html.

Ministerio de Sanidad y Consumo. (2008). Prevención de los problemas derivados del alcohol. $1^{a}$ Conferencia de prevención y promoción de la salud en la práctica clínica en España. Madrid 14 y 15 de junio de 2007. Madrid: Ministerio de Sanidad y Consumo. Recuperado de https://www.mscbs. gob.es/profesionales/saludPublica/prevPromocion/ alcohol/docs/prevencionProblemasAlcohol.pdf.

Monk, R. L. y Heim, D. (2013). A critical systematic review of alcohol-related outcome expectancies. Substance Use and Misuse, 48, 539-557. doi:10.3109/10826084.2013.78 7097.

Moore, G.F., Rothwell, H. y Segrott, J. (2010). An exploratory study of the relationship between parental attitudes and behavior and young people's consumption of alcohol. Substance Abuse Treatment, Prevention, and Policy, 22, 5-6. doi:10.1186/1747-597X-5-6.

Moure-Rodriguez, L., Carbia, C., Lopez-Caneda, E., Corral, M., Cadaveira, F. y Caamaño-Isorna, F. (2018). Trends in alcohol use among young people according to the pattern of consumption on starting university: A 9-year follow-up study. PLoS One, 13, e0193741. doi:10.1371/ journal.pone.0193741.

Moure-Rodríguez, L., Piñeiro, M., Corral, M., Rodríguez-Holguín, S., Cadaveira, F. y Caamaño-Isorna, F. (2016). Identifying predictors and prevalence of alcohol consumption among university students: Nine years of follow-up. PLoS One, 11, e0165514. doi:10.1371/journal. pone.0165514.

National Institute on Alcohol Abuse and Alcoholism. (2004). NIAAA council approves definition of binge drinking. NIAAA Newsletter,3,3. Recuperado de http://pubs. 
niaaa.nih.gov/publications/Newsletter/winter2004/ Newsletter_Number3.pdf.

Newbury-Birch, D., Walker, J., Avery, L., Beyer, F., Brown, N., Jackson, K., ... Stewart, S. (2009). Impact of Alcohol Consumption on Young People: A systematic review of published reviews. Newcastle: Institute of Health and Society at Newcastle university: Department for Children, Schools and Families.

Oei, T. P. y Morawska, A. (2004). A cognitive model of binge drinking: the influence of alcohol expectancies and drinking refusal self-efficacy. Addictive Behaviors, 29, 159179.

Patrick, M. E. y Schulenberg, J. E. (2013). Prevalence and predictors of adolescent alcohol use and binge drinking in the United States. Alcohol Research: Current Reviews, 35, 193-200.

Patrick, M. E., Terry-McElrath, Y. M., Lanza, S. T., Jager, J., Schulenberg, J. E. y O’Malley, P. M. (2019). Shifting age of peak binge drinking prevalence: historical changes in normative trajectories among young adults aged 18 to 30. Alcoholism, Clinical and Experimental Research, 43, 287298. doi:10.1111/acer.13933.

Quinn, P. D. y Fromme, K. (2011). Alcohol use and related problems among college students and their noncollege peers: the competing roles of personality and peer influence. Journal of Studies on Alcohol and Drugs, 72, 622632. doi:10.15288/jsad.2011.72.622.

Robinson, E., Jones, A., Christiansen, P. y Field, M. (2015). Drinking like everyone else: trait self-control moderates the association between peer and personal heavy episodic drinking. Substance Use and Misuse, 50, 590-597. doi:1 0.3109/10826084.2014.991407.

Sargent, J.D.y Babor, T.F. (2020). The relationship between exposure to alcohol marketing and underage drinking is causal. Journal of Studies on Alcohol and Drugs, 19, 113124.

Saunders, J. B., Aasland, O. G., Babor, T. F., De la Fuente, J. R. y Grant, M. (1993). Development of the alcohol use disorders identification test (AUDIT): WHO collaborative project on early detection of persons with harmful alcohol consumption-II. Addiction, 88, 791-804. doi:10.1111/j.1360-0443.1993.tb02093.x.

Schulenberg, J., O’Malley, P. M., Bachman, J. G., Wadsworth, K. N. y Johnston, L. D. (1996). Getting drunk and growing up: trajectories of frequent binge drinking during the transition to young adulthood. Journal of Studies on Alcohol, 57, 289-304.

Schuler, M. S., Tucker, J. S., Pedersen, E. R. y D'Amico, E. J. (2019). Relative influence of perceived peer and family substance use on adolescent alcohol, cigarette, and marijuana use across middle and high school. Addictive Behaviors, 88, 99-105. doi:10.1016/j.addbeh.2018.08.025.

Scott-Sheldon, L. A., Terry, D. L., Carey, K. B., Garey, L. y Carey, M. P. (2012). Efficacy of expectancy challen- ge interventions to reduce college student drinking: a meta-analytic review. Psychology of Addictive Behaviors, 26, 393-405.

Sellers, C. M., McManama O’Brien, K. H., Hernandez, L. y Spirito, A. (2018). Adolescent alcohol use: The effects of parental knowledge, peer substance use, and peer tolerance of use. Journal of the Society for Social Work and Research, 9, 69-87. doi:10.1086/695809.

Serafini, K. A. y Stewart, D. G. (2015). Perceptions of family alcohol use in a young adult sample. The Yale Journal of Biology and Medicine, 88, 205-209.

Simons-Morton, B., Haynie, D. L., Crump, A. D., Saylor, K. E., Eitel, P. y Yu, K. (1999). Expectancies and other psychosocial factors associated with alcohol use among early adolescent boys and girls. Addictive Behaviors, 24, 229-238.

Simons-Morton, B., Haynie, D., Liu, D., Chaurasia, A., Li, K. y Hingson, R. (2016). The effect of residence, school status, work status, and social influence on the prevalence of alcohol use among emerging adults. Journal of Studies on Alcohol and Drugs, 77, 121-132. doi:10.15288/ jsad.2016.77.121.

Steinberg, L. (2014). Adolescence, (10nd ed.). Boston, MA: McGraw-Hill.

Stormshak, E. A., Comeau, C. A. y Shepard, S. A. (2004). The relative contribution of sibling deviance and peer deviance in the prediction of substance use across middle childhood. Journal of Abnormal Child Psychology, 32, 635-649.

Tuunanen, M., Aalto, M. y Seppä, K. (2007). Binge drinking and its detection among middle-aged men using AUDIT, AUDIT-C and AUDIT-3. Drug and Alcohol Review, 26, 295-299.

Varela, J., Carrera, A., Rial, A., Braña, T. y Osorio, J. (2006). Identificación dos trastornos debidos ao consumo de alcohol. Unha adaptación do AUDIT á poboación galega. Santiago de Compostela: Xunta de Galicia-Consellería de Sanidade-Sergas.

Voogt, C., Beusink, M., Kleinjan, M., Otten, R., Engels, R., Smit, K. y Kuntsche, E. (2017). Alcohol-related cognitions in children (aged 2-10) and how they are shaped by parental alcohol use: A systematic review. Drug and Alcohol Dependence, 177, 277-290. doi:10.1016/j.drugalcdep.2017.04.006.

Weitzman, E.R., Nelson, T.F. y Wechsler, H. (2003). Taking up binge drinking in college: The influences of person, social group, and environment. The Journal of Adolescent Health, 32, 26-35. doi:10.1016/s1054-139x(02)00457-3.

White, H. R., Fleming, C. B., Kim, M. J., Catalano, R. F. y McMorris, B. J. (2008). Identifying two potential mechanisms for changes in alcohol use among college-attending and non-college-attending emerging adults. Developmental Psychology, 44, 1625-1639. doi:10.1037/ a0013855. 
Whiteman, S. D., Jensen, A. C. y Maggs, J. L. (2013). Similarities in adolescent siblings' substance use: Testing competing pathways of influence. Journal of Studies on Alcohol and Drugs, 74,104-113. doi:10.15288/jsad.2013.74.104.

Whiteman, S. D., Jensen, A. C., Mustillo, S. A. y Maggs, J. L. (2016). Understanding sibling influence on adolescents' alcohol use: Social and cognitive pathways. Addictive Behaviors, 53, 1-6. doi:10.1016/j.addbeh.2015.09.007.

Wicki, M., Kuntsche, E. y Gmel, G. (2010). Drinking at European universities? A review of students' alcohol use. Addictive Behaviors, 35, 913-924. doi:10.1016/j.addbeh.2010.06.015.

Willett, W. C., Sacks, F., Trichopoulou, A., Drescher, G., Ferro-Luzzi, A., Helsing, E. y Trichopoulos, D. (1995). Mediterranean diet pyramid: a cultural model for healthy eating. The American Journal of Clinical Nutrition, 61, 1402-1406. doi:10.1093/ajcn/61.6.1402S.
Wilsnack, R. W., Wilsnack, S. C. y Obot, I. S. (2005). Why study gender, alcohol and culture?. En I. S. Obot y R. Room (Eds), Alcohol, gender and drinking problems: Perspectives from low- and middle income countries (pp. 1-23). Geneva: World Health Organization.

Windle, M., Haardörfer, R., Lloyd, S. A., Foster, B. y Berg, C. J. (2017). Social influences on college student use of tobacco products, alcohol, and marijuana. Substance Use and Misuse, 52, 1111-1119. doi:10.1080/10826084.2017. 1290116.

Wood, M. D., Read, J. P., Mitchell, R. E. y Brand, N. H. (2004). Do parents still matter? Parent and peer influences on alcohol involvement among recent high school graduates. Psychology of Addictive Behaviors, 18, 19-30. doi:10.1037/0893-164X.18.1.19.

World Health Organization. (2018). Global status report on alcohol and health 2018. Geneva: WHO. 